\title{
EL ANÁLISIS JURÍDICO Y ECONÓMICO INSTITUCIONAL
}

Luis Fernando León Quiroga

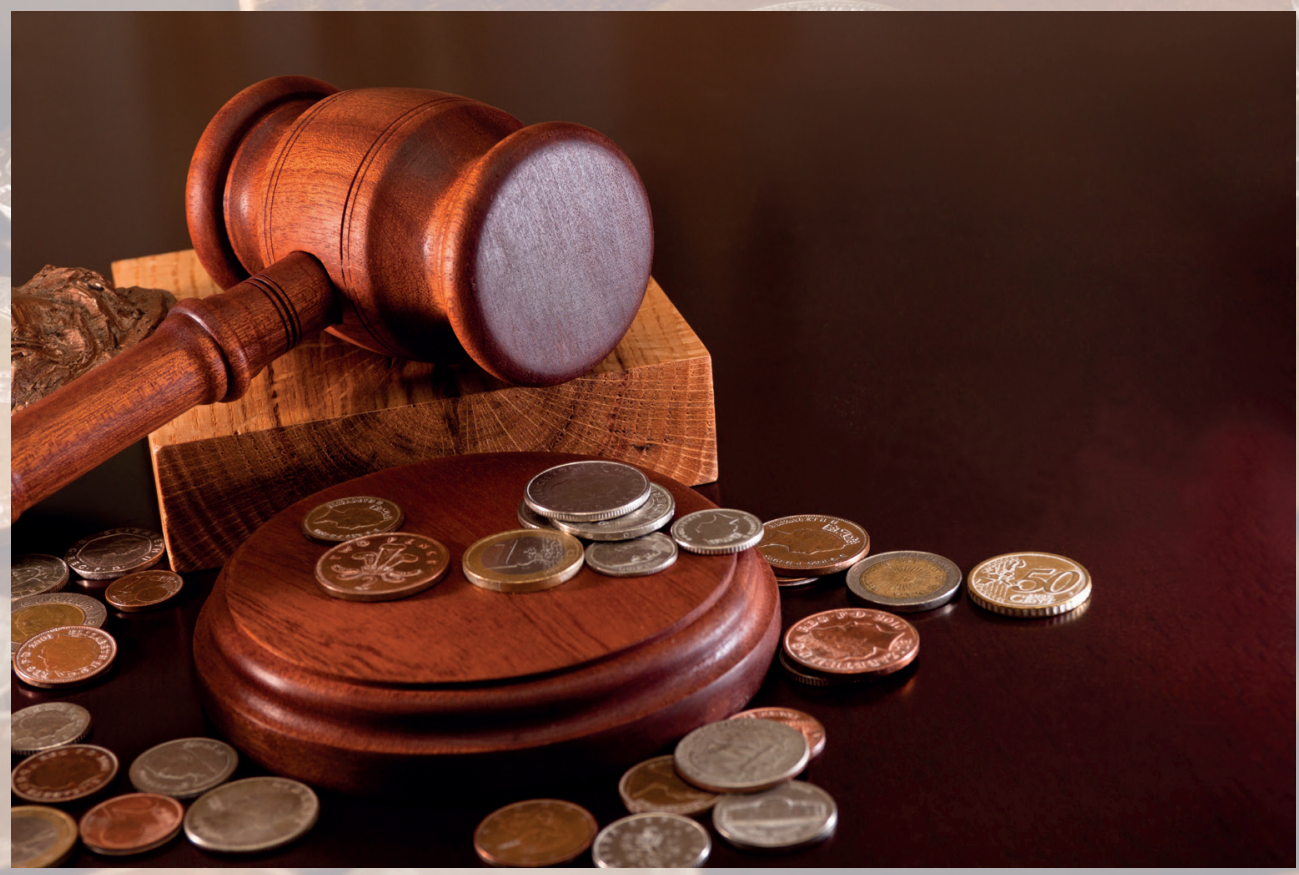





\title{
EL ANÁLISIS JURÍDICO Y ECONÓMICO INSTITUCIONAL"
}

\author{
Luis Fernando León Quiroga \\ Universidad del Rosario, Bogotá D. C., Colombia
}

\section{Resumen}

El análisis de fenómenos sociales se realiza desde diferentes perspectivas: el derecho, la economía, la sociología, la política, la psicología, entre otras disciplinas. Cada una, con una metodología o forma de aproximación más o menos diferenciada para aprehender alguna de sus dimensiones. El propósito de este proyecto es evaluar la conveniencia de adoptar una aproximación interdisciplinaria de economía y derecho, con un enfoque neoinstitucionalista, para analizar un fenómeno social complejo. Con todo, busco justificar la implementación del análisis jurídico y económico (AJE) en lugar del análisis económico del derecho (AED). La diferencia principal yace en que el AED es una disciplina aplicada propiamente desde la economía y el AJE comprende la implementación paritaria de estas dos disciplinas con menor rigurosidad, pero mayor alcance. Para avanzar en esta proposición, se abordará brevemente la noción de propiedad a partir de las herramientas del análisis institucional y de la teoría jurídica moderna de sistemas. Como resultado, fue posible hacer un esquema comprensivo de las diferentes dimensiones de la propiedad en la ciencia económica y la ciencia jurídica, y así justificar el uso del análisis jurídico y económico.

Palabras clave: análisis económico del derecho, derecho económico, neoinstitucionalismo, propiedad, posesión, derecho privado, derecho público.

El autor: luisf.leon@urosario.edu.co

Recibido: 26 de julio 2020; Evaluado: 14 de septiembre 2020; Aceptado: 11 de octubre 2020

Este artículo es producto de la investigación adelantada en la Maestría en Derecho Privado de la Universidad del Rosario. 


\title{
LEGAL AND ECONOMIC INSTITUTIONAL ANALYSIS
}

\author{
Luis Fernando León Quiroga \\ Universidad del Rosario, Bogotá D. C., Colombia
}

\begin{abstract}
The analysis of complex social phenomena is undertaken through various perspectives: law, economics, sociology, politics, psychology, among others. Each one has a specific, more or less differentiated, methodology or approach to grasp the dimensions of said phenomena. Hence, the purpose of this article is to assess the convenience of using an interdisciplinary approach that combines law and institutional economics as a tool of analysis. I use the term "law and economics" instead of "economic analysis of law," as the latter is a discipline applied inherently by economists, while the former approach implies the equal implementation of these two disciplines with less rigor, but greater a scope. To substantiate this claim, the article briefly considers the notion of property using the tools of institutional analysis and modern legal theory of systems. As a result, the article makes a comprehensive outline of the essential aspects of property within economic and legal science, as well as justifies the use of the law and economics approach.
\end{abstract}

Keywords: economic analysis of law, economic law, neo-institutionalism, property, possession, private law, public law.

Author: luisf.leon@urosario.edu.co

Received: July 26, 2020; Reviewed: September 14, 2020; Accepted: October 11, 2020 


\title{
A ANÁLISE JURÍDICA E ECONÔMICA INSTITUCIONAL
}

\author{
Luis Fernando León Quiroga \\ Universidad del Rosario, Bogotá D. C., Colombia
}

\section{Resumo}

A análise de fenômenos sociais é realizada a partir de diferentes perspectivas: o direito, a economia, a sociologia, a política, a psicologia, entre outras disciplinas. Cada uma delas, com uma metodologia ou forma de aproximação mais ou menos diferenciada para apreender algumas de suas dimensões. O propósito deste projeto é avaliar a conveniência de adotar uma aproximação interdisciplinar entre economia e direito, com uma abordagem neoinstitucionalista, para analisar um fenômeno social complexo. Com isso, busco justificar a implementação da "análise jurídica e econômica" (AJE) em vez da "análise econômica do direito" (AED). A principal diferença se encontra no fato de que a AED é uma disciplina aplicada propriamente a partir da economia e a AJE compreende a implementação paritária dessas duas disciplinas com menor rigorosidade, mas com maior alcance. Para avançar nesta proposição, a noção de propriedade será abordada brevemente a partir das ferramentas da análise institucional e da teoria jurídica moderna de sistemas. Como resultado, foi possível criar um esquema compreensivo das diferentes dimensões da propriedade na ciência econômica e na ciência jurídica, e assim justificar o uso da análise jurídica e econômica.

Palavras-chave: análise econômica do direito, direito econômico, neoinstitucionalismo, propriedade, possessão, direito privado, direito público.

O autor: luisf.leon@urosario.edu.co

Recebido: 26 de julho de 2019; avaliado: 14 de setembro de 2020; aprovado: 11 de outubro de 2020. 


\section{Introducción}

La perspectiva es lo que no permite ver a los ciegos ${ }^{1}$. Este podría ser el caso de las ciencias sociales cuando se aproximan a un fenómeno social complejo. Así mismo, apunta al argumento más fuerte a favor de la interdisciplinariedad en las ciencias sociales y su relación dinámica en la academia. Para comprender adecuadamente un objeto de estudio, resulta provechoso, y frecuentemente necesario, valerse de ciencias complementarias.

Con el anterior horizonte presente, pareciera que el análisis económico del derecho (AED) surgiera para poder abordar con mayor rigurosidad y practicidad los fenómenos sociales que interesan a los ciudadanos como agentes económicos y políticos. Esta categoría ha sido utilizada de forma generalizada para referirse a la aplicación de herramientas de la economía al análisis de problemas jurídicos o al derecho mismo. Con todo, los análisis se enmarcan, por lo general, en una dimensión positiva o una dimensión normativa, incluyendo consideraciones de orden ético y técnico ${ }^{2}$. Por lo anterior, se propondrá la categoría del análisis jurídico y económico (AJE) como una disciplina más comprensiva.

El problema por analizar es que las relaciones sociales estudiadas se desenvuelven, normalmente, bajo una amalgama de instituciones formales e instituciones informales que las dotan de sentido y realizan sus efectos. Por lo anterior, conviene adoptar como metodología las categorías del neoinstitucionalismo económico para estudiar adecuadamente los fenómenos sociales complejos. Como método de investigación, se acudió a consulta doctrinaria nacional e internacional sobre las disciplinas mencionadas.

Este texto presentará brevemente la aproximación que hay entre 1) el neoinstitucionalismo económico y la teoría jurídica; 2) la posición del análisis económico del derecho frente al análisis jurídico y económico institucional; 3) aspectos generales del modelo general del AJE y su realización, y 4) las conclusiones generales.

George Christoph Lichtenberg, "Aforismo 300". En Aforismos, editado por George Christoph Lichtenberg y Juan del Solar (Barcelona: Edhasa, 1991), 93.

2 Íñigo Ortiz de Urbina Gimeno, "El análisis Económico del Derecho". En Observar la ley: ensayos sobre metodología de la investigación jurídica (Madrid: Editorial Trotta, 2010), 331-34. 


\section{Nuevo institucionalismo y teoría jurídica}

Esta sección pone en relación la nueva economía institucional (A) con la teoría jurídica moderna (B).

\section{A. La nueva economía institucional y el análisis de instituciones}

La economía institucional puede considerarse como una familia de teorías que comparten la tesis de que las instituciones cuentan en el estudio de la economía; ellas constituyen un objeto esencial de la reflexión. Estas teorías institucionalistas se distinguen de las demás teorías económicas, pues el análisis institucional correspondería más a disciplinas como la ciencia política, la sociología o la historia ${ }^{3}$. Con todo, esta noción clásica de institucionalismo fue desplazada por el nuevo institucionalismo económico (NIE). Dicha disciplina se ocupa de estudiar las instituciones y cómo estas interactúan con arreglos organizacionales. Uno de los ejes importantes de dicha aproximación pretende remover la ficción de los mercados libres de fricciones al incluir las instituciones; así mismo, procura cambiar la economía neoclásica desde una perspectiva estática a una dinámica ${ }^{4}$.

De forma general, las instituciones son acuerdos sociales, cuyo fin comprende reducir los costos de oportunidad — transacción- entre los agentes ${ }^{5}$. Por una parte, estos acuerdos sociales procuran reducir las imperfecciones de la información compartida en un mercado determinado, materializada en la seguridad jurídica y en la predictibilidad de los valores esperados de sus inversiones productivas ${ }^{6}$. Por otra, los costos de transacción representan la pérdida asociada con el proceso de negociación y realización de un intercambio presente o futuro ${ }^{7}$. Los costos de transacción pueden ser vistos como la otra cara de la propiedad: si estos son en exceso elevados, no hay lugar a la distribución de recursos o a la necesidad de la definición de una propiedad específica ${ }^{8}$.

Bernard Chavance, Léconomie Institutionnelle (Paros: La Découverte, 2007), 3.

4 Claude Ménard y Mary M. Shirley, eds. Handbook of new institutional economics (Dordrecht: Springer, 2005), $1-5$.

5 Douglas C. North, "Institutions". Journal of Economic Perspectives, vol. 5 (1991): 97.

6 Douglas C. North, Institutions, institutional change, and economic performance. The Political economy of institutions and decisions (Cambridge, New York: Cambridge University Press, 1990).

7 Walter Nicholson y Christopher Snyder, Intermediate microeconomics and its applications. 11th ed. (Australia: South-Western Cengage Learning, 2010), 375.

8 Douglas W. Allen, "Property rights, transaction costs, and coase: One more time". En Coasean Economics: Law and economics and the new institutional economics, editado por Steven G. Medema. Recent Economic Thought Series 60. (Boston Dordrecht London: Kluwer Academic Publishers, 1998), 109. 
Por un lado, las instituciones pueden ser vistas como un sistema autosuficiente de creencias compartidas sobre cómo se desenvuelven interacciones estratégicas sociales ${ }^{9}$. Estas pueden involucrar reglas provenientes de autoridades — enforcer — ${ }^{10}$ dotadas de poder coercitivo (instituciones formales) o ser acuerdos sociales de conducta generalizados (instituciones informales) que guían el actuar de los integrantes de una comunidad ${ }^{11}$. Su nacimiento y continuidad pueden estar condicionados a su aptitud para reducir costos de transacción — como pareciere ser el caso de los derechos de propiedad — ${ }^{12}$, o bien para reducir costos de administración en el caso de las firmas ${ }^{13}$.

Para Williamson, por otro lado, las instituciones varían de forma asincrónica en el tiempo, reconociendo una suerte de diferenciación por niveles de análisis social ${ }^{14}$. (L1 - instituciones informales) las costumbres, tradiciones y normas religiosas; (L2 - marco institucional) normas referentes a la propiedad privada, la política pública, reglas de solución de conflictos (judicialmente) y burocracia; (L3 - gobernanza) transacciones bajo lineamientos gubernamentales — como los acuerdos entre particulares para internalizar costos— ${ }^{15}$; (L4 - distribución de recursos) instituciones de mercado y de adjudicación de recursos. El tiempo requerido para que haya un cambio estructural — véase, una innovación — disminuye a medida que se desciende en los niveles de análisis social. A pesar de esto, existe una relación bidireccional en la que, como lo expresa Aoki, se refuerzan y modifican mutuamente.

Por lo anterior, para el caso del análisis jurídico y económico de un fenómeno social, conviene analizar el rol de las instituciones formales en la definición, sustento y delimitación de las relaciones en el mercado. A saber, el funcionamiento de la ley lato sensu implica la participación del Estado —enforcer — y comprende los arreglos privados. Así mismo, requiere la interacción sostenida entre agentes, cortes, el sistema administrativo y el aparato legislativo. Inclusive, desempeña un rol principal en la

9 Masahiko Aoki, Toward a comparative institutional analysis. Comparative institutional analysis 2 (Cambridge, Mass: MIT Press, 2001), 26.

10 El sentido de este anglicismo comprende al Estado como garante de los acuerdos entre particulares y el derecho. Se denomina "Estado coactivo" sin que esto implique el uso de la fuerza.

11 North, "Institutions", 97.

12 Harold Demsetz, "Toward a theory of property rights", The American Economic Review, vol. 57, n. 2 (1967): 350.

13 R. H. Coase, "The nature of the firm", Economica, vol. 4, n. ${ }^{\circ} 16$ (1937): 403. https://doi.org/10.2307/2626876

14 Oliver E. Williamson, "The new institucional economics: Taking stock, looking ahead", Journal of Economic Perspectives, vol. XXXVIII (2000): 597.

15 R. H. Coase, "The problem of social cost", The Journal of Law \& Economics, vol. 3 (1960): 1-44. 
organización económica y social, al interactuar/definir los derechos de propiedad, los términos de intercambio y las formas asociativas —firmas_ ${ }^{16}$.

Finalmente, es necesario precisar el sentido de la palabra "norma" como institución relevante que se refiere a un acto o hecho jurídicamente relevante. Para una primera aproximación, las normas son "términos sinónimos de reglas de derecho, de regla jurídica, obligatoria, general e impersonal"17. De forma más particular, este concepto comprende que, en su sentido jurídico: "presuponen el medio de la escritura y, sobre todo, la oración (escrita). Por tanto, se habla de 'proposiciones jurídicas' (Rechtssätze) en vez de 'reglas' o 'normas jurídicas' [...] A lo mejor se podría distinguir $[. .$.$] entre cuatro tipos importantes de proposiciones jurídicas:$ cláusulas contractuales, reglas de derecho, normas en forma de decisiones judiciales y leyes"18. Con todo, en el lenguaje corriente, y de forma relevante, puede sobrepasar una calificación eminentemente jurídica o ligada con el Estado.

La palabra "norma" es utilizada de forma ambigua. Puede denotar un comportamiento social normal, o bien puede referirse a comportamientos promocionados o sancionados por el Estado. Sin embargo, en un sentido lato, estas pueden diferenciarse a partir del sujeto que ejerce control sobre su ejecución. Por lo tanto, la clasificación reconocería variaciones en función de la primera persona (ética personal), segunda persona (contratos) y tercera persona (normas, reglas de organizaciones y la ley en sentido lato $)^{19}$. Así mismo, aún es necesario entender su funcionamiento interno en términos de coexistencia, colisión (para el caso de las reglas) y ponderación (para el caso de los principios) ${ }^{20}$.

No todas estas posibilidades, expresadas como proposiciones, tienen una sanción asociada; sin embargo, deben ser tenidas en cuenta para el entendimiento de la actividad humana, porque componen la estructura institucional formal e informal que ordena la actividad de los agentes. Es en este sistema que la complejidad de los fenómenos sociales puede ser internalizada dentro del análisis del investigador social.

16 Simon Deakin, David Gindis, Geoffrey M. Hodgson, Kainan Huang y Pistor Katharina, "Legal Institutionalism: Capitalism and the constitutive role of law", Journal of Comparative Economics, vol. 45 (2017): 188-200.

17 Serge Guinchard y Jean-Luc Albert, eds., Lexique des termes juridiques. 23. éd., 2015-2016 (Paris: Dalloz, 2015), 705.

18 Thomas Vesting, Teoría del derecho (Bogotá: Universidad Externado de Colombia, 2018). cap. 2 I. A.

19 Robert C. Ellickson, Order without law: how neighbors settle disputes (Cambridge, Mass: Harvard University Press, 1991), 126-31.

20 Robert Alexy, Ensayos sobre la teoría de los principios y el juicio de proporcionalidad (Lima: Palestra Editores, 2019), 20-37. 
Restaría indagar sobre la aproximación institucional que pudiere darse en la ciencia jurídica.

\section{B. Las instituciones en la teoría jurídica: neoiuspositivismo y la teoría de sistemas}

La teoría del derecho también se ha aproximado del análisis institucional para comprender el surgimiento, desarrollo y transformación de sistemas jurídicos. T. Vesting señala que, en principio, un "sistema jurídico" designa la totalidad del derecho ordenado por principios, cuyas oraciones jurídicas e instituciones comprenden una unidad interna. Esta unidad está dada por la organización específica que tengan sus proposiciones y la relación que tengan entre sí a manera de jerarquía/escalonada. La búsqueda de coherencia y plenitud del sistema escalonado se materializaría con el Código Civil francés y la codificación alemana del BGB. En desarrollo de lo anterior, se buscaría un sistema libre de contradicciones y lagunas ${ }^{21}$.

Para Vesting, después de 1800, la ciencia jurídica tomaría la forma de ciencia autónoma de derecho positivo, fundamentada en el libre albedrío del individuo. Con todo, hacia 1900, el modelo sistemático del positivismo jurídico entró en crisis. El cambio fundamental introducido por Hans Kelsen sería desplazar la base del sistema jurídico — previamente en el libre albedrío— hacia una norma fundamental abstracta y científica. La teoría del derecho quedaría reducida a una teoría sobre la naturaleza de las normas y su validez. La relativización de la jerarquía entre la norma jurídica y su aplicación se realizó en un modelo que no distingue de forma tajante la creación del derecho. Se abrió el paso a las indeterminaciones en el sistema de derecho y a criterios extralegales en la estructuración del sistema ${ }^{22}$.

La nueva economía institucional tomaría las instituciones formales del derecho como elementos exógenos y con cierto grado de variabilidad. Con todo, la naturaleza de las instituciones formales examinada presupone una integración institucional coherente que determina incentivos para los agentes económicos. Sin embargo, esta estructura institucional analizada pareciere ser aquella construida por el neoiuspositivismo del siglo XX, que comprende unidad normativa y una norma fundamental para poder operar con éxito. Sin embargo, las teorías modernas del

\footnotetext{
21 Vesting, Teoría del derecho, cap. 3 A-C

22 Ibíd., cap. 3 A-C.
} 
derecho avanzan hacia la construcción de un modelo fundamentado en la teoría de sistemas de la sociología.

La teoría de sistemas, adoptada del sociólogo Niklas Luhmann, para la ciencia jurídica comprende que un sistema es una distinción entre sí y su entorno. Es decir, un sistema jurídico comprende todo lo que es derecho y el entorno es todo lo que no es. Siendo así, se debe identificar qué es derecho —una institución formal específica - de lo que no lo es — una institución informal u otro tipo de fenómeno social一, para luego poder dotarlo de una definición específica dentro de su categoría. No hay, entonces, un criterio de unidad propio a las instituciones formales del derecho ${ }^{23}$. Siendo así, el sistema jurídico comprende un entramado institucional con características particulares. Aunado a lo anterior, el objeto ya no es la norma como un ente diferenciado, sino que se enfoca sobre el lenguaje con relevancia jurídica.

Para Vesting, en primer lugar, el sistema jurídico es caracterizado por la autopoiesis. Esto es, la autoproducción y reproducción de las estructuras y los elementos propios del sistema. La producción dentro del sistema jurídico está limitada por su propia estructura y la diferenciación hecha frente al entorno — lo que no es derecho-. De esta manera, todos los actos y sucesos lingüísticos con relevancia jurídica pertenecerían al sistema jurídico, y todo aquello que no pertenezca al lenguaje propio del sistema hará parte de su entorno ${ }^{24}$. La mutabilidad del sistema estaría dada por el proceso de reflejo e integración de los propios elementos del sistema sobre su entorno.

En particular, las palabras jurídicamente relevantes — los términos jurídicos—están caracterizadas por ser portadoras de un sentido o significado específico. Al ser unidades significativas, constituyen una entidad lingüística diferenciada y autónoma que produce un sentido por sí misma. Así, tienen un sentido jurídico definido que es otorgado por el derecho. De igual forma, bastaría con el uso corriente establecido entre los protagonistas del derecho para que un término adquiera un sentido jurídico diferente de aquel del lenguaje corriente, sustrayéndolo al entorno e incluyéndolo en el sistema de derecho. Así, el vocabulario jurídico es el conjunto de palabras que tienen, a lo menos, un sentido jurídico ${ }^{25}$.

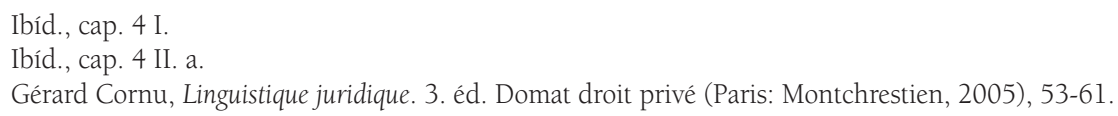


De forma coherente con la evolución de las instituciones propuesta por Williamson y por Aoki, el sistema jurídico, dentro de la teoría de sistemas, tiene un carácter dinámico. Los elementos jurídicamente relevantes pueden ser definidos como una "operación", en el entendido de que son la reproducción temporal de algún elemento relevante en un punto en el tiempo determinado. El tiempo dota de sentido a los elementos del sistema ${ }^{26}$. Así como la significación de propiedad no es la misma entre derecho y economía, el sentido de la propiedad no es el mismo actualmente que en 1887, cuando fue expedido el código civil.

Aunado a lo anterior, los sistemas dentro de los cuales se encuentran las instituciones operan como redes de comunicación para su reproducción de forma recursiva. Dichas comunicaciones y lenguaje tienen una significación jurídica específica que la diferencia de las comunicaciones económicas. La identificación de lo que es derecho y de lo que no es operaría como un código binario ${ }^{27}$.

El sistema, a partir de su propio código binario de identificación del derecho, puede copiar o reflejar el entorno —-no derecho- - dentro de su estructura, de acuerdo con las posibilidades de su comunicación. La reproducción de sus propias estructuras (autorreferencia) para reflejar el entorno (heterorreferencia) constituye su capacidad de incorporar elementos nuevos. A saber: ningún factor foráneo podría ingresar al sistema jurídico si no es por medio del código, las leyes, las normas jurídicas o los precedentes. Las estructuras abiertas en la formulación normativa - como en el derecho de daños- permiten el ingreso de convenciones sociales que normalmente son extrañas al derecho ${ }^{28}$.

Finalmente, la estructuración institucional dentro del sistema jurídico tiene una jerarquía compleja. El paradigma neoiuspositivista, observado por otras disciplinas, asume una estructura normativa jerárquica con un punto de cierre o norma fundamental. Concretamente, puede ilustrarse esta perspectiva con la organización escalonada del sistema jurídico atribuible a Hans Kelsen. En la cúspide se ubicaría la norma fundamental y básica (Grundnorm) que implicaría y condicionaría todas las reglas del sistema ${ }^{29}$.

Vesting, Teoría del derecho, cap. 4 II. b.

Ibíd., cap. 4 II. c.

Ibíd., cap. 4 II. d.

9 Hans Kelsen, Teoría pura del derecho. Serie G: Estudios Doctrinales 20 (México: Universidad Nacional Autónoma de México, 1982), 206. 
Esta abstracción permite racionalizar el sistema jurídico y sus instituciones dentro de una organización coherente y libre de lagunas. Sin embargo, los sistemas jurídicos no operan de esta manera. La producción normativa, la fuente de significado y la aplicación jurídicas no atienden a una única norma fundamental. Por una parte, esta situación puede manifestarse, de forma sencilla, con los "choques de trenes" entre altas cortes en Colombia ${ }^{30}$. Por otra parte, puede ser considerada en las cláusulas abiertas del sistema jurídico y la relación del orden jurídico internacional con el orden jurídico interno de cada país. Si bien el ordenamiento jurídico cuenta con una cláusula de cierre explícita referida en el art. 4 de la Constitución, la Corte Constitucional está en la cabeza de la jurisdicción constitucional, pero comparte el control constitucional con el Consejo de Estado ${ }^{31}$. El funcionamiento complejo de la estructura institucional contraría la concepción jerarquizada del ordenamiento jurídico.

De acuerdo con lo anterior, es posible enmarcar el funcionamiento complejo de la estructura institucional dentro de la teoría de sistemas referida por Vesting. Estos sistemas jurídicos no funcionan como una escalera o pirámide, sino que operan de manera "heterárquica", es decir, de forma colateral o por redes de vecindad; no hay una única norma base que dota de coherencia a todo el sistema, sino que hay diferentes centros de producción de normas y de significación de proposiciones y actos de relevancia jurídica. Este sistema ya no funcionaría a través del método inductivo y deductivo del paradigma iuspositivista ${ }^{32}$; sino que, por el contrario, lo sustituye por el entrelazamiento dinámico, recursivo y horizontal de creación y justificación paralela y constante del derecho ${ }^{33}$. Así mismo, este sistema de derecho puede ser analizado junto con otros sistemas de control social que no pertenezcan propiamente al derecho ${ }^{34}$.

30 Por ejemplo, la diferencia en la posición entre el Consejo de Estado y la Corte Constitucional sobre los efectos de la acción de simple nulidad del art. 84 y 136-1 del Código de Procedimiento Administrativo y de lo Contencioso Administrativo (CPACA) y la seguridad jurídica. En las sentencias C-426 de 2002, T-836 de 2004 y C259 de 2015 impuso la posición contraria en defensa del derecho a la seguridad jurídica. Como segundo ejemplo, la diferencia interpretativa entre la Corte Constitucional y la Corte Suprema de Justicia sobre la posibilidad de prescripción de bienes baldíos. (Sentencias T-844/2014, T-293/2016, T-548/2016, T-407/2017 de la Corte Constitucional; y las decisiones STC-1776 de 2016 y STC943 de 2018 de la Corte Suprema de Justicia).

31 La Corte Suprema de Justicia es el máximo tribunal de la jurisdicción Ordinaria de Colombia, Constitución Política de Colombia art. 234., el Consejo de Estado lo es para lo contencioso administrativo.

32 "[...] las normas jurídicas ya no se sitúan en un nivel superior y categóricamente distinto del de su aplicación como en el iuspositivismo y sus sistemas." Vesting, Teoría del derecho, cap. 4 III. a. par. 2.

33 Ibíd., cap. 4 III. a.

34 Ellickson, Order without law, 123-26. 
La modelación institucional de un sistema jurídico que opera con redes de vecindad sin una jerarquía estricta captura de mejor manera la realidad del funcionamiento complejo del ordenamiento jurídico ${ }^{35}$. Si bien no hay una sustitución total de la metodología del iuspositivismo, conviene adoptar el diseño de un sistema heterárquico para orientar adecuadamente el análisis de la ciencia jurídica y de la ciencia económica. La internalización de los criterios de la teoría jurídica, en particular de la teoría de sistemas, en el análisis de la economía institucional enriquecería el análisis comprensivo de los fenómenos sociales abordados por la economía y el derecho.

Habida cuenta de lo anterior, conviene integrar la nueva economía institucional y de la teoría de sistemas en el análisis jurídico y económico.

\section{El problema: AED vs. AJE}

Esta sección enfrenta el análisis económico del derecho (AED) y el análisis jurídico y económico (AJE) (A), seguido del acercamiento entre economía y derecho (B).

\section{A. El análisis económico del derecho}

El análisis económico del derecho, por mucho tiempo fue relacionado con el análisis económico de las normas antimonopolísticas, de las normas tributarias y de la utilidad pública. Posteriormente, se transformó en la aplicación de los métodos empíricos y las teorías de la ciencia económica a los sistemas legales y sus diferentes ramas ${ }^{36}$. El inicio de esta disciplina podría ubicarse en los estudios de Bentham, entre 1789 y 1830, en lo que respecta a las medidas de bienestar social y el utilitarismo. Posteriormente, Coase (1960), Becker (1968), Calabresi (1970) y Posner (1972) definieron las bases sobre las cuales ha prosperado dicha disciplina ${ }^{37}$. A saber: el análisis neoinstitucional coaseano de la economía construye sobre las bases metodológicas del AED y enriquece el análisis económico de la normatividad legal ${ }^{38}$.

35 Vesting, Teoría del derecho, cap. 4 III. b.

36 Richard A. Posner, Economic analysis of law. 7th ed. (New York, NY: Wolters Kluwer Law \& Business: Aspen Publishers, 2007), 19.

37 Steven Shavell, Foundations of economic analysis of law (Cambridge, Mass: Belknap Press of Harvard University Press, 2004), 1666.

38 Steven G. Medema, ed., Coasean economics: Law and economics and the new institutional economics. Recent Economic Thought Series 60 (Boston, Dordrecht, London: Kluwer Academic Publishers, 1998). 
Las herramientas económicas comprenden nociones fundamentales, entre ellas: 1) la relación inversa entre el precio de los bienes y la cantidad demandada; 2) la teoría de la decisión racional de los agentes y la maximización de los beneficios, y 3) la cantidad de recursos y su adjudicación tienden a encontrar su uso más valioso cuando pueden ser tranzados en un mercado competitivo. Así mismo, tienen definiciones particulares sobre utilidad, valor y eficiencia ${ }^{39}$.

Una definición general la comprende como "la aproximación al derecho que se basa en la aplicación de técnicas de análisis económico al estudio de los fenómenos e instituciones jurídicas"40. De igual forma, lo han definido como aquella "nueva mirada acerca del Derecho, a partir de la economía, de la que se utiliza leyes, principios, metodología e instituciones, que proyecta sobre materiales u objetos propios del Derecho [...]. En este contexto se emplean técnicas analíticas que visualizan las soluciones a controversias jurídicas como elecciones sociales que implican sopesar costos y beneficios o ponderar valores sociales en conflicto [...] gira en torno a la noción de eficiencia o bienestar agregado, al considerar que las normas jurídicas pueden ser entendidas como intentos institucionales de maximizar el bienestar agregado"41.

En particular, el análisis económico del derecho se ocupa de la regulación del mercado (teoría de los monopolios, regulación del empleo, utilidad pública), las normas comerciales sobre sociedades y mercados financieros, la distribución de los ingresos y la riqueza, el procedimiento legal —legislativo y litigioso-, el constitucionalismo, y temas relacionados, como la responsabilidad por accidentes, el derecho de la propiedad, el derecho de los contratos, el litigio civil y la ejecución coactiva de la ley.

De la anterior definición, podemos extraer que el análisis económico del derecho es una labor realizada por un economista sobre el derecho o sus problemas. El sujeto activo de este estudio es el economista que toma como objeto de estudio instituciones jurídicas por su eficiencia, o bien cuyo interés es encontrar soluciones alternativas a problemas que tuvieron origen en la teoría jurídica. Por lo tanto, más allá de ser una práctica que involucre un diálogo interdisciplinario, es la aproximación unívoca

Posner, Economic analysis of law, 3-26.

Ortíz de Urbina, "Observar la ley", 323.

${ }^{41}$ El AED pretende responder dos preguntas: ¿cuáles son los efectos de las reglas legales en el comportamiento de actores relevantes? Y ¿cuáles son los efectos socialmente deseables de las reglas legales? Shavell, Foundations of economic analysis of law, 1666. Kluger, Análisis económico del derecho, 9. 
de las herramientas de la economía sobre las herramientas del derecho o sobre los resultados de esta última. En consecuencia, el análisis económico del derecho no debería ser conceptualizado como la unión de las dos prácticas, sino como una rama especializada de la economía institucional que se preocupa específicamente por el derecho o por sus soluciones.

Siendo así, el análisis económico del derecho no es aquella rama que mezcla economía y derecho para abordar un fenómeno social complejo. Es, en realidad, una práctica económica especializada sobre otra disciplina. Lo anterior no desestima los avances teóricos que han sido realizados bajo esta consigna. Por el contrario, considero necesario hacer esta precisión terminológica para orientar el sentido de este artículo y poner en relieve los alcances del estudio que expondré en el texto. En particular, no se adelantará una investigación fruto del "análisis jurídico del derecho", sino que corresponde al análisis jurídico y económico (AJE) o análisis jurídico y económico institucional.

A pesar de parecer sinónimos (AED y AJE), conllevan diferencias sustanciales. En primer lugar, debe darse importancia al orden de las palabras y su sentido en conjunto. El AED privilegia la contribución de la economía al estudio jurídico. La parte activa del estudio sería el economista frente a la parte pasiva (el derecho). Por el contrario, el "análisis jurídico y económico" pareciera que las ubica sobre un mismo pie de relevancia, con un poco más de preponderancia del análisis jurídico ${ }^{42}$. El sujeto activo del análisis sería el investigador, tendría como herramientas la teoría económica y la teoría jurídica, y como objeto, un fenómeno social específico.

Así, el análisis jurídico y económico introduce detalles institucionales en el alcance del análisis económico del derecho. Esto dificulta la labor de análisis, pero puede ser más enriquecedor y revelador ${ }^{43}$. El AED se inclina sobre la ciencia jurídica como objeto de estudio. El AJE, al igual que las ciencias de las cuales se vale, tiene como objeto un fenómeno social específico.

Por lo anterior, podría proponerse como definición que el análisis jurídico y económico es la labor investigativa que se vale de las herramientas de la economía y el estudio detallado de las instituciones de un sistema jurídico, aplicando sus

42 Geoffrey P. Miller, "Law and economics versus economic analysis of law", American Bankruptcy Institute Law Review, n. ${ }^{\circ} 2$ (2011): 459.

43 Miller, 465 
categorías, para evaluar el entramado institucional que rodea a un fenómeno social complejo y así poder hacer recomendaciones y valoraciones.

Habida cuenta de lo anterior, conviene abordar los puntos de encuentro entre las mencionadas disciplinas.

\section{B. Análisis, economía y derecho}

La economía podría ser entendida formalmente como el "estudio de la destinación de recursos escasos entre usos alternativos" ${ }^{\prime 4}$. Por su parte, el derecho, en sentido general, es "el conjunto de reglas tendientes a organizar la conducta del hombre en sociedad y cuyo respeto está asegurado por el poder público. El derecho objetivo reconoce y sanciona por sí mismo los derechos subjetivos, entendidas como prerrogativas atribuidas en su interés a los individuos, que les permite gozar de una cosa, de un valor o de exigir de otro una prestación" ${ }^{\prime 5}$.

En particular, la microeconomía comprende el estudio de "las decisiones económicas de los individuos y las firmas, y cómo estas decisiones crean mercados"46. De forma coherente, el derecho ${ }^{47}$ civil puede ser definido como "el conjunto de reglas de derecho normalmente aplicables en las relaciones de personas privadas entre ellas [...] En un sentido más general, puede representar la totalidad del derecho privado", y el derecho comercial como "el conjunto de reglas jurídicas aplicables a los comerciantes en el ejercicio de su actividad profesional y aplicable también, aunque de manera más excepcional, a la actividad comercial"48.

De las dos aproximaciones anteriores, es posible identificar al menos un común denominador: relaciones entre agentes de un contenido transaccional. Este es el fenómeno social complejo que abordan ambas disciplinas valiéndose de herramientas pretendidamente propias. Sin embargo, la evaluación económica de las relaciones

44 Nicholson y Snyder, Intermediate microeconomics and its applications, 38.

45 Guinchard y Albert, Lexique des termes juridiques, 389.

46 Nicholson y Snyder, Intermediate microeconomics and its applications, 38.

47 Elijo de forma deliberada las nociones de derecho privado y comercial, porque i) en la tradición jurídica continental es el noyau esencial de su estudio; ii) guardan coherencia con la definición expuesta de microeconomía y economía, y iii) serán pertinentes para el desarrollo del modelo de este escrito. Sin embargo, el AJE también puede ser utilizado para analizar fenómenos de relevancia para el derecho público.

48 Guinchard y Albert, Lexique des termes juridiques, 391-312. 
entre agentes supone un entramado institucional previo a la relación de su objeto de estudio. Es decir, presupone una rule of law sobre la cual construir un modelo ${ }^{49}$.

De la misma manera, la teoría jurídica define el significado objetivo de los acontecimientos, no por su facticidad, sino porque una norma se ha referido a ellos $^{50}$. La producción de la norma que dota de significado objetivo a las actuaciones y los acontecimientos es fruto de un proceso legislativo - ley en sentido latojudicial — sentencias—, ejecutivo —actos administrativos - o bien privado — contratos- que traen tras de sí consideraciones de orden político, económico, social o inclusive irracional.

En consecuencia, a pesar de la clara diferenciación entre las dos ciencias sociales, ambas pueden recurrir, directa o indirectamente, a herramientas de otras disciplinas para poder abordar un fenómeno social de la forma más completa posible. No podríamos, entonces, hablar propiamente de una teoría pura del derecho o una teoría pura de la economía. Ambas teorías se ocuparían de un fenómeno social relevante, como lo son las relaciones transaccionales entre personas. Harían uso de sus herramientas inmediatas — para la economía, la microeconomía; para el derecho, la teoría jurídica del derecho privado—, y utilizarían de forma indirecta o nutrirían su análisis de la otra disciplina.

Por ejemplo, la microeconomía, de forma general, supone un entramado institucional que garantiza transacciones como la propiedad privada, su protección y libre disposición. Por su parte, el derecho fija sus reglas teniendo en consideración criterios económicos y políticos, como en el caso de la protección de accionistas minoritarios, la definición del momento para declarar patrimonio y la forma de su cobro.

Landreth y Colander señalan que la ciencia de la economía, por lo general, no incluye explícitamente las instituciones por el simple hecho de que el análisis institucional es complejo y la búsqueda científica pretende encontrar relaciones subyacentes de forma elegante que encajen en las técnicas existentes. Evadir el análisis explícito de instituciones, sin embargo, no libera a la ciencia económica de ellas: la economía

\footnotetext{
49 La dicotomía entre economía "normativa" — el deber ser — y economía "positiva" — lo que es— refleja esta cercanía a las ciencias jurídicas que se ocupan del "deber ser". Sola, "Metodología de la economía positiva, los límites de la predicción."

50 Kelsen, Teoría pura del derecho, 17.
} 
neoclásica incluye una variedad de supuestos sobre las instituciones en su modelo estructural subyacente ${ }^{51}$.

Habiendo mostrado sus características más importantes, resulta conveniente pasar a indagar en la estructuración de un modelo metodológico que integre las diferentes aproximaciones.

\section{El modelo: metodología y aplicación}

Esta sección aborda la representación de un modelo y herramientas de análisis jurídico y económico institucional (A), y desarrolla las conclusiones de su implementación (B).

\section{A. El fenómeno social a la luz de la economía y el derecho}

Siendo así, para ilustrar esta relación interdisciplinaria, podría ubicarse en un extremo de una línea a la teoría jurídica pura y, en el otro extremo, a la teoría económica pura. En el centro equidistante se ubicaría el fenómeno social complejo que ambas ciencias sociales pretenden estudiar. Ubicarse en un extremo significa que el investigador se vale únicamente de lo que se considerarían herramientas puras o exclusivas de una de las ciencias. La perspectiva goza de perfecta rigurosidad, mas su análisis está tan alejado de la realidad como sus herramientas se lo permiten.

A medida que se recorre la recta hacia el punto central en el cual se ubica el fenómeno social, se pierde rigurosidad o pertinencia en la ciencia de la que se aleja, pero adquiriría un conocimiento del fenómeno social complejo más comprensivo que el que se tendría en las abstracciones de los extremos. Con todo, dicha pérdida de rigurosidad reduce la información procesable que se obtenga del estudio y puede disminuir la fiabilidad de los resultados.

No obstante, esta representación gráfica no realiza juicios de valor sobre el punto en el cual el investigador decida ubicarse para estructurar su estudio. Es más, la representación bidimensional sugeriría que el uso igualitario de las dos ciencias permitiría conocer en la mayor medida de lo posible un fenómeno complejo. Esta conclusión, aunque se sigue de los argumentos expuestos anteriormente, es falsa, puesto que no es una representación perfecta o adecuada de la forma como funcionan

51 Harry Landreth y David C. Colander, History of economic thought. 4th ed. (Boston: Houghton Mifflin, 2002), 508. 
las ciencias sociales. Es únicamente un modelo que permite ilustrar, de forma más o menos adecuada, una relación entre tres elementos específicos: la economía, el derecho y el fenómeno social. Así, excluye de forma deliberada la relación que hay con otras ramas del conocimiento, el estado del arte de cada una, la evolución del lenguaje a través del tiempo, el cambio de paradigmas científicos y el peso de las consideraciones de cada una.

Como todo modelo en economía, es una representación de la realidad que procura incluir únicamente los elementos importantes, definiendo factores exógenos entre presupuestos axiológicos — como la institución de la propiedad — y con indiferencia consciente de otros elementos - exclusión de irracionalidad o información imperfecta—, sin que esto signifique una pérdida relevante de precisión en la apreciación que quiere realizarse. Si consideráramos más de dos disciplinas, la representación del modelo sería de forma geoide y tendría al fenómeno social objeto de estudio en su núcleo. Sin embargo, debido a que esta investigación está acotada por las herramientas de la ciencia económica y del derecho, es más conveniente representar su relación en un modelo bidimensional.

Como anota T. Vesting: "[... ] una perspectiva limitada sobre el mundo tiene también ventajas significativas. El ciego se da cuenta de que el bastón bien manejado facilita una buena orientación y garantiza una gran sensibilidad para el entorno. Precisamente a causa del cerramiento del sistema y el uso de tan solo una distinción (estable/no estable) es fácil reconocer patrones repetidos y, por tanto, diseñar un 'mapa' más o menos complejo el entorno"52.

Habida cuenta de lo anterior, la noción de aproximación entre las ciencias económicas y jurídicas, que contiene el modelo anteriormente descrito, puede ilustrarse de manera simple con la representación de la diferencia fundamental en el sentido de un elemento esencial a ambas disciplinas: la propiedad. En particular, esta noción es inevitable en la economía y transversal al derecho. Para la primera, la distribución eficiente de recursos escasos la supone; para la segunda, las relaciones entre personas tienen connotaciones y consecuencias patrimoniales para el derecho público y privado ${ }^{53}$.

Vesting et al., Teoría del derecho, cap. 4 II. d. par. 4.

53 Este mismo análisis podría ser realizado para los conceptos de contrato, transacción, ejecución, entre otros. Sin embargo, encuentro que la propiedad está en el centro. 
Para la economía, esta es la noción nuclear que da sentido a la adjudicación de recursos escasos y es el concepto sobre el cual se estructura el pensamiento económico convencional. En el caso del derecho, es posible identificar en el Código Civil colombiano — que adoptó en un inicio la estructura del Código ${ }^{54}$ traducido por Andrés Bell $0^{55}$ - una estructura centrada en el manejo de la propiedad. En particular, el artículo primero define que "El Código Civil comprende disposiciones legales sustantivas que determinan especialmente los derechos de los particulares, por razón del estado de las personas, de sus bienes, obligaciones, contratos y acciones civiles" (cursivas mías). Los derechos de los particulares aquí mencionados pueden ligarse de forma directa con exigencias de contenido económico, o bien con situaciones que tendrán consecuencias valorables económicamente.

Seguido al título preliminar, el Código se divide en libros sobre: i) las personas, ii) de los bienes y su dominio, posesión, uso y goce; iii) la sucesión por causa de muerte y las donaciones entre vivos; y iv) de las obligaciones en general y de los contratos. Desde una perspectiva centrada en la propiedad, los libros corresponden a: i) quién puede tener propiedad, en qué forma, qué sucede con el patrimonio cuando hay un matrimonio y qué expectativas hay entre el núcleo familiar sobre este y su disposición; ii) qué puede ser apropiable y de qué manera se puede disponer de ello; iii) qué sucede con la propiedad cuando el titular muere, y iv) reglas generales sobre los negocios. Con esto presente, puede abordarse su sentido bajo una perspectiva sistemática.

En primer lugar, para el derecho, de forma general y clásica, podría definirse la propiedad como: "un derecho real principal que confiere a su titular, el propietario, todas las prerrogativas sobre el bien, objeto de su derecho; tradicionalmente se distinguen tres prerrogativas: el 'usus', el 'abusus' y el 'fructus'. El derecho de la propiedad, por sí mismo, constituye un bien. Éste es imprescriptible. Sin embargo, para algunos autores, no comprende un bien, sino la relación de exclusividad que existe entre cada bien y la persona a la cual le pertenece" ${ }^{156}$. De esta definición puede

54 La codificación como fruto de la Revolución francesa trae el culto a la ley. Remplazar las costumbres francesas por una ley general que tuviere indirectamente la voluntad popular marca un cambio importante en la fuente de la legitimidad de la norma y su relación con el pueblo. Gilles Cuniberti, Grands systèmes de droit contemporains. 2. ed. (Paris: L.G.D.J., Lextenso Éd, 2011), 44, 45.

55 Andrés Botero Bernal, "El código civil de Andrés Bello y el movimiento exegético en Colombia", Comparative Law Review, vol. 9, n. ${ }^{\circ} 1$ (2018): 157-63.

56 Guinchard y Albert, Lexique des termes juridiques, 397. 
extraerse la naturaleza de la propiedad (derecho), lo que otorga (uso, disposición y goce $)^{57}$, el sujeto activo (el titular) y frente a quién se hace valer (la sociedad) ${ }^{58}$.

Desde una perspectiva económica, podría decirse que "los derechos de propiedad son instrumentos de la sociedad y derivan su razón de ser del hecho de que ayudan a los hombres a formarse aquellas expectativas que le permiten razonablemente tener tratos con otros. Estas expectativas encuentran su expresión en leyes, costumbres y la moral de la sociedad. El titular de los derechos de propiedad posee el consentimiento de sus semejantes para permitirle actuar de formas particulares. Es importante anotar que los derechos de propiedad contienen el derecho de beneficiar o hacer daño a uno mismo o a otros"

De forma particular, para la ciencia económica, el concepto-rol-naturaleza de la propiedad no es el centro de discusión, como sí lo es en la ciencia jurídica. Las definiciones son sucintas y refieren generalmente la materialización del derecho de propiedad; a saber, su comercialidad y vocación a ser depósito de valor; los elementos de modo, tiempo y lugar en que esta se entiende realizada, y las consecuencias de su disposición.

La definición prima facie también comprende, en líneas generales, la titularidad sobre algo, su vocación de comercialidad, la noción de derecho y la oponibilidad a la sociedad. Sin embargo, el sentido de derecho de propiedad económica se acerca más a la posesión que a la propiedad para el análisis jurídico.

\section{B. El modelo del análisis jurídico y económico del derecho}

En el análisis económico, la noción de derecho atiende al control de facto sobre un bien, sin ahondar si tiene respaldo legal o es ejecutable por una institución estatal. La posesión ha sido usada "para referirse al control o uso posible de un activo o recurso, con indiferencia de cualquier derecho asumido o decretado que lo permita [...] La posesión se refiere al control físico de activos, material o incorpóreos, sin un título formal sobre ellos" ${ }^{\prime 60}$. De acuerdo con lo anterior, la propiedad, en sentido

\footnotetext{
57 Es posible conferir derechos de propiedad limitados, como en el caso del leasing. Geoffrey M. Hodgson, "Much of the 'economics of property rights' devalues property and legal rights", Journal of Institutional Economics, vol 11, n. ${ }^{\circ} 4$ (2015): 689. https://doi.org/10.1017/S1744137414000630".

58 El art. 669 del Código Civil Colombiano.

59 Demsetz, "Toward a Theory of Property Rights", 347

60 Hodgson, "Much of the 'economics of property rights' devalues property and legal rights", 687.
} 
económico, está intrínsecamente ligada con la tenencia ${ }^{61}$. Relacionado con esto, la perspectiva económica sustentada en la posesión puede englobar transacciones entre agentes económicos en el marco de lo legal, lo extralegal y lo ilícito.

Por el contrario, la noción de propiedad en sentido jurídico está intrínsecamente ligada con la presencia de un Estado, con una regla de derecho, que justifica la tenencia y la defiende coercitivamente. El derecho de propiedad legal "no simplemente adiciona una justificación normativa para tener alguna cosa: a través de las interacciones sociales que involucran a la autoridad y las expectativas también refuerza la habilidad de facto de usar y retener un activo" 62 . Inclusive, el criterio para determinar la propiedad, a diferencia de la noción económica ligada con la posesión, no yace en la tenencia, sino en el respaldo estatal.

Esto es evidente en el hecho de que es posible ser propietario sin la tenencia o el control del bien, como sucede en el caso de la nuda propiedad o en el hecho en que el titular de un bien pueda requerir al poseedor su restitución ${ }^{63}$. Este tipo de propiedad requiere un esquema institucional que la respalde (instituciones notariales y registrales), que decida las disputas entre pretendidos titulares (rama judicial a través de la acción reivindicatoria ${ }^{64}$ y que garantice la ejecución de la solución de controversias (poder ejecutivo y policivo).

Geoffrey M. Hodgson considera que, en consecuencia, lo que podría considerarse como "derecho de propiedad" en el análisis económico, es en realidad una noción laxa de posesión ${ }^{65}$ para la jurisprudencia. De igual forma, lo que es "derecho legal de propiedad" referiría el derecho de propiedad de la ciencia jurídica. La propiedad no puede ser, entonces, una mera relación entre un bien y un objeto, o entre un bien y la ciudadanía. La propiedad se refiere directamente al derecho de un titular o titulares, reconocidos formalmente por una autoridad, para explotar sus bienes o disponer de ellos por venta u otros medios. La diferencia crucial yace entonces en

${ }^{61}$ Por el contrario, la definición de derechos de propiedad de la economía neoinstitucionalista comprende que los derechos de propiedad son la habilidad propia, sin sanción aplicable, de ejercer una decisión sobre un bien, servicio o persona.

62 Hodgson, "Much of the 'economics of property rights' devalues property and legal rights", 690.

63 El art. 384 del Código General del proceso define el proceso de restitución de bien inmueble arrendado al dueño que ha otorgado su uso y usufructo a un tercero. Colombia, Código General del Proceso.

64 Guinchard y Albert, Lexique des termes juridiques, 927.

65 El art. 762 del Código Civil colombiano la define: "La posesión es la tenencia de una cosa determinada con ánimo de señor o dueño, sea que el dueño o el que se da por tal, tenga la cosa por sí mismo, o por otra persona que la tenga en lugar y a nombre de él." República de Colombia, Código Civil. 
la adjudicación de derechos formales por la autoridad ${ }^{66}$. Por ello, la propiedad, en su sentido más verdadero, tiene otro requisito: la autoridad política del Estado ${ }^{67}$. Antes del Estado, solo hay posesión ${ }^{68}$.

La perspectiva anterior encuentra que la vigencia de las normas, y por consiguiente la legitimidad de la propiedad, depende principalmente de la fuerza del Estado. Esto se sobrepone a la idea de derecho consuetudinario y a las convenciones sociales que se relacionan de forma estrecha con él. De igual forma, la costumbre, como fuente de derecho, queda relegada ante la idea iuspositivista del Estado legislador y el Estado ejecutor. Los fenómenos de codificación en Europa continental, y la consolidación del Estado nacional y de la sociedad industrial al final del siglo XIX definieron que la creación del derecho es un dominio del Estado con su poder de mando ${ }^{69}$.

La noción de la ejecución estatal centra la legitimidad de dicha acción en un único ente ejecutor. Sin embargo, como fue mencionado en la sección de teoría de sistemas, es posible que el centro de decisión y ejecución no sea único, sino que, por el contrario, haya diferentes fuentes de autoridad que puedan contrariarse. Esto sucede porque, en realidad, se está frente a un sistema heterárquico de fuentes de derecho que permite la producción de elementos normativos en apariencia incompatibles, como lo son decisiones judiciales disonantes sobre un mismo tema.

Así como el respaldo y la ejecución de los derechos de propiedad es dinámica, en tanto que depende de su realización en el tiempo, el contenido de la propiedad en su dimensión de derecho también sigue la misma suerte. Es decir, las transacciones pueden realizarse sobre activos tangibles o intangibles actuales, o bien sobre su expectativa. De igual forma, la realización del derecho depende del entramado institucional, formal e informal, que realicen de forma efectiva su traslado de titularidad —o desmembramiento de la propiedad— por la vía consensual o bien por la vía litigiosa.

\footnotetext{
${ }^{66}$ La definición de Barzel es similar: "Legal rights are the claims over assets delineated by the state as the property of particular individuals or institutions". Yoram Barzel, A theory of the state: economic rights, legal rights, and the scope of the state. Political economy of institutions and decisions. (Cambridge, New York: Cambridge University Press, 2002), 157.

67 Sobre el carácter coactivo del Estado. Kelsen, Teoría Pura del Derecho, 46, 47.

68 Hodgson, "Much of the 'economics of property rights' devalues property and legal rights", 687-89.

69 Vesting, Teoría del derecho, cap. 5.
} 
Por lo anterior, el derecho de propiedad tiene una naturaleza dinámica, no solo por el hecho de la disposición de su estado actual o expectativa, sino, también, por la operatividad del sistema jurídico y el proceso de creación y reproducción interna de sus elementos jurídicamente relevantes. En consecuencia, sería conveniente incluir un factor de incertidumbre derivado de la mutabilidad del sistema jurídico y de la incertidumbre que rodea la decisión de los órganos de ejecutivos y judiciales, diferencias entre órganos de igual jerarquía y reversibilidad de las decisiones. Dicho factor debe tenerse en cuenta junto con las expectativas de volatilidad del valor de los activos.

Por consiguiente, a través del AJE puede acotarse adecuadamente la noción en su contexto bajo una perspectiva neoinstitucionalista y de teoría jurídica en el análisis jurídico y económico de la propiedad. Puede evaluarse la relación directa de los sujetos con el bien y con el resto de la sociedad al hacer una radiografía de sus elementos esenciales (usus, frutus y abusus), el sistema institucional jurídico que gobierna las transacciones (ordenamiento jurídico), la diferenciación binaria de lo que es derecho — propiedad-y de lo que no lo es — posesión—, el entorno (ilegalidad y extrajuridicidad) por contraposición a lo jurídico, la internalización de factores dinámicos referentes al bien apropiado (volatilidad sobre su precio y cantidad, expectativa de pago o defraudación), así como del sistema (incertidumbre jurídica y multiplicidad de soluciones en un sistema heterárquico).

\section{Conclusiones}

Por un lado, la nueva economía institucional es la rama heterodoxa de la economía que se ocupa de indagar sobre los efectos de las instituciones en el comportamiento de los agentes y los efectos de la definición estructural específica de un esquema institucional formal o informal. Por otro lado, la teoría jurídica se ocuparía de desarrollar herramientas analíticas normativas — escuela iuspositivista alemana o bien escuela analítica anglosajona-, integrando componentes de la sociología del derecho — como lo es la teoría de sistemas - ${ }^{70}$. Ambas disciplinas abordan fenómenos sociales complejos, y su utilización conjunta se encuentra en el análisis jurídico y económico institucional. Por contraposición, el análisis económico del derecho supone una aproximación económica a las normas legales en sentido lato. Esto implica que el ejercicio investigativo está acotado por las herramientas de la economía.

70 Vesting, cap. 1 I. a, b, c. 
Por una parte, el AED pertenecería directamente a la economía, en cuanto comprende el uso de los principios y el razonamiento de la economía para entender las normas formales. Este objeto de estudio sería el derecho o la teoría jurídica visto a la luz de la teoría de la decisión racional, la métrica de la eficiencia económica, el diseño de modelos de interacción estratégica, entre otros. Por otra parte, el AJE comprende una colaboración genuina entre ambas disciplinas: la economía añadiría la percepción enriquecida de la ciencia económica con las herramientas clásicas mencionadas y el análisis enriquecido del neoinstitucionalismo; la teoría jurídica, por su parte, sumaría su entendimiento de instituciones complejas, con su política, ejecución y dinámicas socialmente relevantes ${ }^{71}$.

No obstante, privilegiar el uso del AJE sobre el AED conllevaría una pérdida de rigurosidad, en tanto que, para mantenerse "enraizado en la realidad" y abordar propiamente las complejidades de las relaciones sociales, habría dificultades en la modelación propia del análisis económico. Con todo, esto daría la posibilidad de tener un abanico de posibilidades considerablemente mayor para poder explicar adecuadamente el fenómeno social objeto de investigación ${ }^{72}$. Para mostrarlo, se abordó brevemente la noción de la propiedad desde la perspectiva de la economía y el análisis de la teoría de sistemas, con el fin de identificar las incompatibilidades entre las nociones estudiadas en cada ciencia, los puntos de tangencia y las intersecciones entre cada aproximación disciplinaria.

Todo lo anterior procura incentivar la investigación en análisis jurídico y económico, al mostrar brevemente sus capacidades y limitaciones. Resta extender y profundizar este análisis propuesto frente a fenómenos sociales relevantes como el consumo.

\section{Bibliografía}

Alexy, Robert. Ensayos sobre la teoría de los principios y el juicio de proporcionalidad. Lima: Palestra Editores, 2019.

Allen, Douglas W. "Property rights, transaction costs, and coase: One more time". En Coasean Economics: Law and economics and the new institutional economics, editado por Steven G. Medema. Recent Economic Thought Series 60. Boston Dordrecht London: Kluwer Academic Publishers, 1998.

71 Miller, "Law and Economics versus Economic Analysis of Law", 460.

72 Ibíd., 470. 
Aoki, Masahiko. Toward a comparative institutional analysis. Comparative institutional analysis 2. Cambridge, Mass: MIT Press, 2001.

Barzel, Yoram. A theory of the state: economic rights, legal rights, and the scope of the state. Political economy of institutions and decisions. Cambridge, New York: Cambridge University Press, 2002.

Botero Bernal, Andrés. "El código civil de Andrés Bello y el movimiento exegético en Colombia”. Comparative Law Review, vol. 9, n. ${ }^{\circ} 1$ (2018): 155-74.

Chavance, Bernard. Léconomie Institutionnelle. Paris: La Découverte, 2007.

Coase, R. H. "The Nature of the Firm". Economica, vol. 4, n. ${ }^{\circ} 16$ (1937): 386-405. https:// doi.org/10.2307/2626876.

- "The Problem of Social Cost". The Journal of Law E Economics, vol. 3 (1960): 1-44.

Colombia. Código General del Proceso, Pub. L. No. Ley 1564 de 2012 de Colombia (2012). http://www.secretariasenado.gov.co/senado/basedoc/ley_1564_2012.html.

. Constitución Política de Colombia, Pub. L. No. 116 de 1991 de Colombia (1991).

Cornu, Gérard. Linguistique juridique. 3. éd. Domat droit privé. Paris: Montchrestien, 2005.

Cuniberti, Gilles. Grands systèmes de droit contemporains. 2. éd. Paris: L.G.D.J., Lextenso Éd, 2011.

Deakin, Simon, David Gindis, Geoffrey M. Hodgson, Kainan Huang, y Pistor Katharina. "Legal Institutionalism: Capitalism and the constitutive role of law". Journal of Comparative Economics, vol. 45 (2017): 188-200.

Demsetz, Harold. "Toward a theory of property rights". The American Economic Review, vol. 57, n. ${ }^{\circ} 2$ (1967): 347-59.

Ellickson, Robert C. Order without law: how neighbors settle disputes. Cambridge, Mass: Harvard University Press, 1991.

Guinchard, Serge y Jean-Luc Albert, eds. Lexique des termes juridiques. 23. éd., 2015-2016. Paris: Dalloz, 2015.

Hodgson, Geoffrey M. "Much of the 'economics of property rights' devalues property and legal rights". Journal of Institutional Economics, vol. 11, n. ${ }^{\circ} 4$ (2015): 683-709. https:// doi.org/10.1017/S1744137414000630.

Ortiz de Urbina Gimeno, Íñigo. "El análisis económico del derecho". En Observar la ley: ensayos sobre metodología de la investigación jurídica. Madrid; Editorial Trotta, S.A., 2010.

Kelsen, Hans. Teoría pura del derecho. Serie G: Estudios Doctrinales 20. México: Universidad Nacional Autónoma de México, 1982.

Kluger, Viviana, ed. Análisis económico del derecho. 1. ed. Buenos Aires: Heliasta, 2006.

Landreth, Harry y David C. Colander. History of economic thought. 4th ed. Boston: Houghton Mifflin, 2002.

Lichtenberg, Georg Christoph, y Juan del Solar. Aforismos. 3. reimpr. Barcelona: Edhasa, 1991. 
Medema, Steven G., ed. Coasean economics: Law and economics and the new institutional economics. Recent Economic Thought Series 60. Boston Dordrecht London: Kluwer Academic Publishers, 1998.

Ménard, Claude y Mary M. Shirley, eds. Handbook of new institutional economics. Dordrecht: Springer, 2005.

Miller, Geoffrey P. "Law and economics versus economic analysis of law". American Bankruptcy Institute Law Review, n. ${ }^{\circ}$ Issue 2 (2011): 459.

Nicholson, Walter y Christopher Snyder. Intermediate microeconomics and its applications. 11th ed. Australia, Mason, OH: South-Western Cengage Learning, 2010.

North, Douglas. C. "Institutions". Journal of Economic Perspectives, vol. 5 (1991): 91-112. - Institutions, institutional change, and economic performance. The Political economy of institutions and decisions. Cambridge, New York: Cambridge University Press, 1990.

Posner, Richard A. Economic analysis of law. 7th ed. New York, NY: Wolters Kluwer Law \& Business: Aspen Publishers, 2007.

República de Colombia. Código Civil, Pub. L. No. Ley 84 de 1873. Accedido 23 de febrero de 2020. http://www.secretariasenado.gov.co/senado/basedoc/codigo_civil.html.

Shavell, Steven. Foundations of economic analysis of law. Cambridge, Mass: Belknap Press of Harvard University Press, 2004.

Sola, Juan Vicente. "Metodología de la economía positiva, los límites de la predicción". Revista Libertas, vol. XIII, n. ${ }^{\circ} 45$ (octubre de 2006). https://www.eseade.edu.ar.

Vesting, Thomas. Teoría del derecho. Bogotá: Universidad Externado de Colombia, 2018.

Williamson, Oliver E. "The new institucional economics: Taking stock, looking ahead". Journal of Economic Perspectives, vol. XXXVIII (2000): 595-613. 